\title{
Relativistic particle dynamics-Lagrangian proof of the no-interaction theorem
}

\author{
G. Marmo and N. Mukunda* \\ Istituto di Fisica Teorica, Universita di Napoli, Napoli, Italy \\ and Istituto Nazionale di Física Nucleare, Gruppo Teorico, Sezione di Napoli, Napoli, Italy \\ E. C. G. Sudarshan \\ Centerfor Particle Theory, Departmeni of Physics, The University of Texas at Austin, Austin, Texas 78712
}

\begin{abstract}
An economical proof is given, in the Lagrangian framework, of the no-interaction theorem of relativistic particle mechanics. It is based on the assumption that there is a Lagrangian, which if singular is allowed to lead at most to primary first-class constraints. The proof works with Lagrange rather than Poisson brackets, leading to considerable simplifications compared to other proofs.
\end{abstract}

\section{INTRODUCTION}

Classical dynamics contains as an idealization the concept of a spinless and structureless mass point. In the relativistic context this may be identified as an irreducible realization of the Poincare group, the mass of the particle being the only nontrivial Casimir invariant of the realization.' In formulating this point of view one works at the level of the canonical Hamiltonian version of dynamics, making use of the Poisson bracket structure to image the Poincaré Lie algebra and canonical transformations to image group elements. Equally well, one could start with the more elementary Newton-Lagrange notion of a oneparameter family of space-time points describing a straight-line world trajectory with Newton's first law of motion obeyed.

When we pass from a single free particle to a collection of free particles the Hamiltonian description remains valid, with the Poisson bracket realization of the Poincare-Lie algebra now using the sums of the individual particle contributions. One can now ask: can this scheme accommodate interactions, that is, can the particle world lines or space-time trajectories be made to transform properly under frame changes though influencing each other due to mutual interaction?

These two requirements of Poincare invariance and the geometrical world-line transformation property [the world-line condition, (WLC)] are distinct, and together they lead to rather stringent limitations on the permissible dynamics. In fact, in the simplest case of instant-form Hamiltonian dynamics with no constraints they lead to the no-interaction theorem.' A.s in other contexts, so in particle complexes: individual response to change of reference frame by each particle without reference to the overall system destroys any cohesion of the system.

While the original proofs of this theorem were couched in the language of phase space and Poisson brackets, ${ }^{2}$ it is possible to give a Lagrangian proof as well. ${ }^{3}$ This investigation has shown that it is not the phase-space formulation that lies at the basis of the theorem. Rather the real cause resides in the geometrical structures and the con- flitting conditions formulated in terms of them.

It is, of course, possible to evade the no-interaction theorem by extending the relativistic dynamical framework without giving up the physical content of the WLC. ${ }^{4}$ All such models work within the generalized constraintdynamics formalism. They are not intended, in general, to be based on a Lagrangian. These theories contain nontrivial interactions consistent with the WLC. Even the additional requirement of separability can be accommodated.' $\boldsymbol{S o}$ one may naturally suspect that the nointeraction theorem cannot hold in the presence of constraints.

The present investigation addresses this question on the assumption that there is a Lagrangian. We show that if only primary first-class constraints are present in a Lagrangian theory we can still prove the no-interaction theorem. In this case it is not necessary to invoke the constraints explicitly and proceed to a Dirac bracket formalism. It is more direct to prove it in the Lagrangian framework, with the fundamental role played by Lagrange brackets.

The plan of the paper is as follows. In Sec. II we review and expose the structures of Lagrange and Poisson brackets from an intrinsic viewpoint. We use this opportunity to remind ourselves about the natural geometrical distinction between the two (apart from the algebraic relation between matrices of these brackets in the nonsingular cases). We also spell out the geometrical way in which the determination of the dynamics, and the action of the Poincare group, are to be handled. Both these use the language of vector fields on the tangent bundle over configuration space. Since in our treatment the independent parameter in the Lagrangian is physical time, apart from the dynamical vector field the only other truly nontrivial vector fields are those generating Lorentz boosts. Section III deals with the imposition of the WLC: A simple analysis shows that with this condition the Lorentz-boost vector fields are determined completely in terms of the dynamics. Section IV carries through the calculation to show the emergence of the no-interaction theorem. We emphasize the irrelevance of the existence of primary con- 
straints at least as far as establishing the absence of any interaction terms in the Lagrangian. Section $\mathbf{V}$ compares our proof with previous ones and discusses our result in the light of the constraint formulation of interacting systems and the prospects of a Lagrangian existing in such theories.

\section{GEOMETRICAL FRAMEWORK}

\section{A. General considerations}

In dealing with particle dynamics in a geometrical framework, the carrier space is usually identified as either the tangent bundle $T Q$ (Newtonian and Lagrangian formalism) or the cotangent bundle $T^{*} Q$ (Hamiltonian formalism) on the configuration space $Q .{ }^{6}$ While the latter is suited for the study of the "canonical" aspects of dynamics, the former with the Lagrangian formalism is better suited for the expression of relativistic invariance.

From a geometrical point of view the relevance of the cotangent bundle relies on the existence of the natural one-form

$$
8=p_{r} d q^{r}
$$

on it. This is the starting point for the canonical formalism which goes along the following lines. From 8 one derives by exterior differentiation a nondegenerate twoform

$$
\omega=d \theta=d p_{r} \wedge d q^{r} .
$$

This two-form establishes an isomorphism between the set of vector fields on $T^{*} \mathcal{Q}$, namely, $\mathscr{X}\left(T^{*} Q\right)$, and the set of one-forms on $T^{*} Q, \mathscr{Q}^{*}\left(T^{*} Q\right)$ (an elegant way of saying that it allows for raising and lowering indices). This isomorphism leads to an association of a (Hamiltonian) vector field $X_{f} \in \mathscr{Q}\left(T^{*} Q\right)$ with any function $f$ on $T^{*} Q$, as the solution to the algebraic equation

$$
i_{X_{f}} \omega=d f \text {. }
$$

If we denote the (local) coordinates $q^{r}, p_{r}$ of $T^{*} Q$ collectively by $\xi^{\mu}$, and the corresponding components of $\omega$ by $\omega_{\mu \nu}$, we have

$$
\begin{aligned}
& \omega=\frac{1}{2} \omega_{\mu \nu} d \xi^{\mu} \wedge d \xi^{\nu}, \\
& X_{f}=\left(X_{f}\right)^{\mu} \frac{\partial}{\partial \xi^{\mu}}, \\
& d f=\frac{\partial f}{\partial \xi^{\mu}} d \xi^{\mu}
\end{aligned}
$$

and the association (2.3)reads

$$
\left(X_{f}\right)^{\mu} \omega_{\mu \nu}=\frac{\partial f}{\partial \xi^{\nu}} \text {. }
$$

The Poisson bracket of two functions $f, g$ is defined by setting

$$
\omega\left(X_{f}, X_{g}\right)=-\{f, g\},
$$

or in coordinates

$$
\{f, g\}=-\omega_{\mu \nu}\left(X_{f}\right)^{\mu}\left(X_{g}\right)^{\nu}
$$

If we introduce the inverse of $\left(\omega_{\mu \nu}\right)$, say $\left(\omega^{\mu \nu}\right)$, we have a more explicit expression

$$
\{f, g\}=\omega^{\mu \nu} \frac{\partial f}{\partial \xi^{\mu}} \frac{\partial g}{\partial \xi^{\nu}} .
$$

From a geometrical point of view the Poisson brackets are naturally associated with a bivector field

$$
\Lambda=\omega^{\mu \nu} \frac{\partial}{\partial \xi^{\mu}} \wedge \frac{\partial}{\partial \xi^{\nu}},
$$

whereas the object that the two-form $\omega$ more naturally leads to is the Lagrange bracket $\omega(X, Y)$ of any two vector fields $X, Y \in \mathscr{X}\left(T^{*} Q\right)$. The definition of the Poisson brackets in Eq. (2.6) in terms of $\omega\left(X_{f}, X_{g}\right)$ uses the wellknown fact that a reciprocity relation connects the two kinds of brackets, with one being the inverse of the other in a suitable sense.

This discussion serves to clarify that it is the Lagrange brackets, rather than the Poisson brackets, that arise immediately from the natural one-form 8 of Eq. (2.1) on $T^{*} Q$. Construction of the Poisson brackets requires the inversion of $\left(\omega_{\mu v}\right) .^{7}$

On the tangent bundle $T Q$, i.e., in the $q \dot{q}$ space, there is no natural one-form. It is possible however to define a one-form on $T Q$ if one is given a Lagrange function $\mathscr{L}$ on $T Q$. This is done by "pulling back" 8 from $T^{*} Q$ to $T Q$ with the replacement $p_{r} \rightarrow \partial \mathscr{L} / \partial \dot{q}^{r}$, i.e.,

$$
\theta_{\mathscr{L}}=\underset{\substack{\partial \\ \partial \dot{L}}}{\partial \dot{q}} d q^{r} .
$$

(In geometrical language one first defines the fiber derivative of the function $\mathscr{L}, F \mathscr{L}: T Q \rightarrow T^{*} Q$, and then pulls back the one-form $\theta$ ) Imitating the passage from 8 to $\omega$ on $T^{*} Q$, we get on $T Q$ the two-form

$$
\begin{aligned}
\omega_{\mathscr{L}}= & d \theta_{\mathscr{L}}=d\left[\frac{\partial \mathscr{L}}{\partial \dot{q}^{r}}\right] \wedge d q^{r} \\
= & \frac{\partial^{2} \mathscr{L}}{\partial \dot{q}^{r} \partial \dot{q}^{s}} d \dot{q}^{s} \wedge d q^{r}+\frac{1}{2}\left[\frac{\partial^{2} \mathscr{L}}{\partial \dot{q}^{r} \partial q^{s}}\right. \\
& \left.\quad-\frac{\partial^{2} \mathscr{L}}{\partial \dot{q}^{s} \partial q^{r}}\right] d q^{s} \wedge d q^{r} .
\end{aligned}
$$

It is clear at this point that $\omega_{\mathscr{L}}$ is nondegenerate (i.e., invertible) if and only if

$$
\operatorname{det}\left[\frac{\partial^{2} \mathscr{L}}{\partial \dot{q}^{r} \partial \dot{q}^{s}}\right] \neq 0
$$

Thus the definition of Poisson brackets among functions on $T Q$ requires this nondegeneracy condition, whereas on the other hand, Lagrange brackets can always be defined among vector fields on $T Q$, once a Lagrange function has been given. It seems then more natural to work exclusively with Lagrange brackets on $T Q$ whenever we want to allow for the possibility that we have a singular Lagrangian leading to a constrained system, because then the condition (2.12)is violated.

Canonical transformations on $T Q$ (with respect to a given $\mathscr{L}$ ) can be defined as those which preserve the 
Lagrange bracket. They need not be lifts to $\boldsymbol{T Q}$ of point transformations on $Q$, but can mix $q$ 's with $\dot{q}$ 's. For an infinitesimal transformation generated by a vector field $X \in \mathscr{P}(T Q)$, the condition to be canonical is

$$
L_{X} \omega_{\mathscr{L}}=0 \text {. }
$$

\section{B. The N-particle problem}

For our specific problem, the independent coordinates of $\boldsymbol{Q}$ will be written as $q_{a j}$, with indices appearing as subscripts. Here, indices $a, b, c, \ldots$ run from 1 to $N$ and serve as particle labels, while $j, k, l, m, \ldots$ go over $1,2,3$ and are Cartesian vector indices. The summation convention on repeated indices will not be used hereafter, and every summation will be explicitly indicated. In using the symbol $\dot{q}_{a y}$ for velocities, the dot specifies the derivative with respect to the physical time of an inertial observer.

We write the Lagrangian as $\mathscr{L}(q, \dot{q})$. The general form of $\omega_{\mathscr{L}}$ in Eq. (2.11), implies, and is equivalent to, the following three relations:

$$
\begin{aligned}
& \omega_{\mathscr{L}}\left(\partial / \partial \dot{q}_{a j}, \partial / \partial \dot{q}_{b k}\right)=0, \\
& \begin{aligned}
\omega_{\mathscr{L}}\left(\partial / \partial \dot{q}_{a j}, \partial / \partial q_{b k}\right) & =\omega_{\mathscr{L}}\left(\partial / \partial \dot{q}_{b k}, \partial / \partial q_{a j}\right) \\
& =\partial^{2} \mathscr{L} / \partial \dot{q}_{a j} \partial \dot{q}_{b k},
\end{aligned} \\
& \begin{aligned}
\omega_{\mathscr{L}}\left(\partial / \partial q_{a j}, \partial / \partial q_{b k}\right) \\
=\frac{1}{2}\left(\partial^{2} \mathscr{L} / \partial q_{a j} \partial \dot{q}_{b k}-\partial^{2} \mathscr{L} / \partial q_{b k} \partial \dot{q}_{a j}\right) .
\end{aligned}
\end{aligned}
$$

The dynamical vector field $\mathbf{A}$, which will have to obey the Euler-Lagrange equations of motion, is an element of $\mathscr{Z}(T Q)$ having the second-order form

$$
\begin{aligned}
& \Delta=\sum_{a} \Delta^{(a)}, \\
& \Delta^{(a)}=\sum_{j}\left(\dot{q}_{a j} \partial / \partial q_{a j}+A_{a j} \partial / \partial \dot{q}_{a j}\right) .
\end{aligned}
$$

We have denoted the accelerations by $A_{a j}$. The equations of motion are expressed as an algebraic condition on $\boldsymbol{A}$ :

$$
\begin{aligned}
& i_{\Delta} \omega_{\mathscr{L}}=-d E_{\mathscr{L}}, \\
& E_{\mathscr{L}}=i_{\Delta} \theta_{\mathscr{L}}-\mathscr{L}, \\
& \theta_{\mathscr{L}}=\sum_{a j} \frac{\partial \mathscr{L}}{\partial \dot{q}_{a j}} d q_{a j} .
\end{aligned}
$$

(Note that $E_{\mathscr{L}}$ does not depend on $A_{a j}$; it is the energy function on $T Q$.)

Our basic assumption is that the Lagrangian $\mathscr{L}$ is either nonsingular or, if it is singular, it does not lead to any secondary constraints, and furthermore it allows Eq. (2.16) to be solved for a second order $\mathrm{A}$ all over $\boldsymbol{T} \boldsymbol{Q}$. In the former case the accelerations $A_{a j}$ are uniquely determined functions on $\boldsymbol{T} \boldsymbol{Q}$ and we have an unambiguous dynamics $\mathbf{A}$ all over $\boldsymbol{T} \boldsymbol{Q}$. In the latter case, solutions $\mathbf{A}$ to Eq. (2.16) do exist at all points of $\boldsymbol{T Q}$ but there is some degree of ambiguity in the solution, namely, up to elements in the vertical kernel of $\omega_{\mathscr{L}}$. But in either case, every point of $\boldsymbol{T Q}$ is allowed to be chosen as a possible initial condition, which is physically necessary for a system of $N$ particles.
Dynamical evolution on $\boldsymbol{T Q}$, generated by $\mathbf{A}$, is obviously canonical since from Eq. (2.16)follows

$$
L_{\Delta} \omega_{\mathscr{L}}=0
$$

We will similarly assume that the entire Lie algebra of the Poincaré group $\mathscr{P}$ is represented by vector fields on $\boldsymbol{T Q}$ obeying Eq. (2.13). Thus we assume the existence of vector fields $\boldsymbol{X}_{P_{j}}, \boldsymbol{X}_{J_{j}}, \boldsymbol{X}_{\boldsymbol{K}_{j}}$ generating spatial translations, spatial rotations, and pure Lorentz transformations, respectively, and obeying

$$
L_{X_{p_{j}}} \omega_{\mathscr{L}}=L_{X_{J}} \omega \mathscr{L}=L_{X_{K} .} \omega_{\mathscr{L}}=0
$$

The set of ten vector fields $\Delta, X_{P_{j}}, X_{J_{j}}, X_{K_{j}}$ must obey commutation relations corresponding to the Lie algebra of $\mathscr{P}$; of all these relations, the only ones we need explicitly in proving the no-interaction theorem are

$$
\left[X_{K_{j}}, \Delta\right]=X_{P_{j}} \text {. }
$$

The forms of $X_{P_{J}}$ and $X_{J_{j}}$ are immediate:*

$$
\begin{aligned}
& X_{P_{j}}=-\sum_{a} \partial / \partial q_{a j}, \\
& X_{J_{j}}=-\sum_{a k l} \epsilon_{j k l}\left[q_{a k} \frac{\partial}{\partial q_{a l}}+\dot{q}_{a k} \frac{\partial}{\partial \dot{q}_{a l}}\right] .
\end{aligned}
$$

The structure of $X_{K_{j}}$ will follow in Sec. III from the WLC.

While translations and rotations have associated vector fields $X_{P_{j}}$ and $X_{J_{j}}$ which have the free-particle form the vector field A for time translations must of nature be different to account for interaction. The question now arises as to the form of the boost vector fields $X_{K_{j}}$. By virtue of the Poincart algebra

$$
\left[X_{K_{j}}, X_{P_{k}}\right]=\delta_{j k} \Delta .
$$

Consequently if $\mathbf{A}$ includes an interaction contribution and $X_{P_{k}}$ does not, than $X_{K_{j}}$ must have an interaction contribution. Hence the particle trajectories cannot transform by the familiar free-particle formulas. The precise form of $X_{K_{j}}$ is determined in Sec. 111. The "Lorentztransformation" law is itself determined by the dynamics.

\section{THE WLC AND THE BOOST GENERATORS}

In the Hamiltonian formalism on $T^{*} Q$, it is well known that a canonical transformation describing a symmetry of a system maps a state at a certain time (the independent parameter) into another state at the same time so as to preserve the equations of motion. For a relativistic system the pure Lorentz or boost generator gives rise to a canonical transformation mapping physical conditions at a certain time in one inertial frame onto physical conditions at the same value of time but in a Lorentztransformed frame. ${ }^{9}$ It is on this basis that, in the instant form of relativistic dynamics, the WLC was originally derived. We express this form of the WLC in the language of $T Q:$ it is then the requirement that 


$$
L_{X_{K}} q_{a k}=q_{a j} \dot{q}_{a k}
$$

We now show that this condition determines $X_{K_{j}}$ in terms of $\boldsymbol{A}$.

Equation (3.1) already fixes the "horizontal" part in $X_{K_{j}}$, namely, the part involving $\partial / \partial q$. To fix the remaining $\partial / \partial \dot{q}$ part, we apply $L_{\Delta}$ to Eq. (3.1), and use the commutation relation (2.19) and the forms of $A$ and $X_{P_{j}}$ :

$$
\begin{aligned}
L_{X_{K},} \dot{q}_{a k} & =L_{X_{K}} L_{\Delta} q_{a k} \\
& =L_{X_{P_{f}}} q_{a k}+L_{\Delta}\left(q_{a j} \dot{q}_{a k}\right) \\
& =-\delta_{j k}+\dot{q}_{a j} \dot{q}_{a k}+q_{a j} A_{a k} .
\end{aligned}
$$

Putting together Eqs. (3.1) and (3.2), we can see that $X_{K_{j}}$ takes the form

$$
X_{K_{j}}=\sum_{a k}\left[q_{a j} \dot{q}_{a k} \frac{\partial}{\partial q_{a k}}+\left(\dot{q}_{a j} \dot{q}_{a k}-\delta_{j k}+q_{a j} A_{a k}\right) \frac{\partial}{\partial \dot{q}_{a k}}\right] \text {. }
$$

This can also be written in terms of the parts $\Delta^{(a)}$ of $A$, Eq. $(2,15)$, as

$$
X_{K_{j}}=\sum_{a} q_{a j} \Delta^{(a)}+\sum_{a k}\left(\dot{q}_{a j} \dot{q}_{a k}-\delta_{j k}\right) \frac{l}{\partial \dot{q}_{a k}} .
$$

So when, and to the extent that the accelerations $A_{a j}$ are determined by the equations of motion (2.16), the boost generator $X_{K_{j}}$ gets determined to the same extent. We may note that in this section, in arriving at the above form for $X_{K_{j}}$, the Lagrange function $\mathscr{L}$ and the two-form $\omega_{\mathscr{L}}$ have not appeared at all.

\section{PROOF OF THE MAIN THEOREM}

Our proof of the no-interaction theorem involves three steps, each of which is quite elementary. We present these steps in sequence.

Step I. Apply $\boldsymbol{L}_{X_{K_{m}}}$ to the identity (2.14a) and use the property (2.18)to get

$$
\begin{aligned}
\omega_{\mathscr{L}}\left[\left[X_{K_{m}}, \frac{\partial}{\partial \dot{q}_{a j}}\right], \frac{\partial}{\partial \dot{q}_{b k}}\right] \\
+\omega_{\mathscr{L}}\left[\frac{\partial}{\partial \dot{q}_{a j}},\left[X_{K_{m}}, \frac{\partial}{\partial \dot{q}_{b k}}\right]\right]=0 .
\end{aligned}
$$

From Eq. (3.3)we find

$$
\left[X_{K_{m}}, \frac{\partial}{\partial \dot{q}_{a j}}\right]=-q_{a m} \frac{\partial}{\partial q_{a j}}+\cdots,
$$

where the dots stand for $\partial / \partial \dot{q}$ terms which, again because of Eq. (2.14a), will not contribute in Eq. (4.1). Therefore Eq. (4.1) simplifies to

$\left(q_{a m}-q_{b m}\right) \omega_{\mathscr{L}}\left[\frac{\partial}{\partial \dot{q}_{a j}}-, \frac{\partial}{\partial q_{b k}}\right]=\left(q_{a m}-q_{b m}\right) \frac{\partial^{2} \mathscr{L}}{\partial \dot{q}_{a j} \partial \dot{q}_{b k}}=0$.
Thus we have obtained some information on the components of $\omega_{\mathscr{L}}$ appearing in Eq. (2.14b), namely, for distinct particles, $a \neq b$ they vanish:

$$
\omega_{\mathscr{L}}\left[\frac{\partial}{\partial q_{a j}}, \frac{\partial}{\partial \dot{q}_{b k}}\right]=0, a \neq b .
$$

Correspondingly we have a decomposition of the Lagrangian with respect to the dependence on the velocities: 10

$$
\mathscr{L}(q ; \dot{q})=\sum_{a} \mathscr{L}^{(\boldsymbol{a})}\left(q ; \dot{q}_{a}\right) .
$$

The ambiguity in the isolation of individual terms $\mathscr{L}^{(\boldsymbol{a})}$ is only to the extent that functions of the q's alone could be reassigned freely.

Step II. To the result of step I we apply first $L_{A}$, then $L_{X_{K_{m}}}$, compare the results, and derive some conclusions concerning the components of $\omega_{\mathscr{L}}$ appearing in Eq. (2.14c). So with $a \neq b$ understood, we apply $L_{\Delta}$ to Eq. (4.4)and use Eq. (2.17)to get

$\omega_{\mathscr{L}}\left[\left[\Delta, \frac{\partial}{\partial q_{a j}}\right], \frac{\partial}{\partial \dot{q}_{b k}}\right]+\omega_{\mathscr{L}}\left[\frac{\partial}{\partial q_{a j}},\left[\Delta, \frac{\partial}{\partial \dot{q}_{b k}}\right]\right]=0$.

The first term here is identically zero because of Eq. (2.14a) and the fact that $\left[\Delta, \partial / \partial q_{a j}\right]$ involves only $\partial / \partial \dot{q}$ terms. For the second term we have

$$
\left[\Delta, \frac{\partial}{\partial \dot{q}_{b k}}\right]=-\frac{\partial}{\partial q_{b k}}-\sum_{c l} \frac{\partial A_{c l}}{\partial \dot{q}_{b k}} \frac{\partial}{\partial \dot{q}_{c l}},
$$

so Eq. (4.6) gives

$$
\omega_{\mathscr{L}}\left[\frac{\partial}{\partial q_{a j}}, \frac{\partial}{\partial q_{b k}}\right]=-\sum_{c l} \frac{\partial A_{c l}}{\partial \dot{q}_{b k}} \omega_{\mathscr{L}}\left[\frac{\partial}{\partial q_{a j}}, \frac{\partial}{\partial \dot{q}_{c l}}\right] .
$$

By Eq. (4.4) resulting from step I, only the term with $c=a$ survives on the right, so application of $L_{\Delta}$ to (4.4) has led to

$$
\begin{aligned}
\omega_{\mathscr{L}}\left[\frac{\partial}{\partial q_{a j}}\right. & \left., \frac{\partial}{\partial q_{b k}}\right) \\
& =-\sum_{l} \frac{\partial A_{a l}}{\partial \dot{q}_{b k}} \omega_{\mathscr{I}}\left[\frac{\partial}{\partial q_{a j}}, \frac{\partial}{\partial \dot{q}_{a l}}\right], a \neq b .
\end{aligned}
$$

Next when we apply $\boldsymbol{L}_{\boldsymbol{X}_{K_{m}}}$ to Eq. (4.4)we get at first, as in Eq. (4.6),

$$
\begin{aligned}
\omega_{\mathscr{L}}\left[\left[X_{K_{m}}, \frac{\partial}{\partial q_{a j}}\right], \frac{\partial}{\partial \dot{q}_{b k}}\right] \\
+\omega_{\mathscr{L}}\left(\frac{\partial}{\partial q_{a j}},\left[X_{K_{m}}, \frac{\partial}{\partial \dot{q}_{b k}}\right]\right)=0 .
\end{aligned}
$$

The first term again vanishes. This time on account of Eq. (4.4); in fact 


$$
\left[X_{K_{m}}, \frac{\partial}{\partial q_{a j}}\right]=-\delta_{j m} \sum_{l} \dot{q}_{a l} \frac{\partial}{\partial q_{a l}}+\cdots,
$$

where the omitted terms are of the form $\partial / \partial \dot{q}$. For the second term in Eq. (4.10) we have

$$
\left[X_{K_{m}}, \frac{\partial}{\partial \dot{q}_{b k}}\right]=-q_{b m} \frac{\partial}{\partial q_{b k}}-\sum_{c l} q_{c m} \frac{\partial A_{c l}}{\partial \dot{q}_{b k}} \frac{\partial}{\partial \dot{q}_{c l}}+\cdots,
$$

where the omitted terms are of the form $\partial / \partial \dot{q}_{b}$ and so drop out in view of Eq. (4.4). We now use Eq. (4.12) in Eq. (4.10), realize that in the sum on $c$ only $c=a$ contributes, and so finally get

$$
\begin{aligned}
q_{b m} \omega_{\mathscr{L}} & {\left[\frac{\partial}{\partial q_{a j}}, \frac{\partial}{\partial q_{b k}}\right] } \\
& =-q_{a m} \sum_{l} \frac{\partial A_{a l}}{\partial \dot{q}_{b k}} \omega_{\mathscr{L}}\left[\frac{\partial}{\partial q_{a j}}, \frac{\partial}{\partial \dot{q}_{a l}}\right], a \neq b .
\end{aligned}
$$

If we compare the results (4.9) and (4.13), we see that

$$
\left(q_{a m}-q_{b m}\right) \omega_{\mathscr{L}}\left[\frac{\partial}{\partial q_{a j}}, \frac{\partial}{\partial q_{b k}}\right]=0 .
$$

Thus application of $L_{\Delta}$ and $L_{X_{K_{m}}}$ to the results of step I yields some information on the components of $\omega_{\mathscr{L}}$ in Eq. (2.14c), namely,

$$
\omega_{\mathscr{L}}\left[\frac{\partial}{\partial q_{a j}}-, \frac{\partial}{\partial q_{b k}}\right]=0, \quad a \neq b .
$$

If we use the already separated form of the Lagrangian in Eq. (4.5) to evaluate these components of $\omega_{\mathscr{L}}$ according to Eq. (2.14c), what we have is

$$
\frac{\partial^{2} \mathscr{L}^{(a)}\left(q ; \dot{q}_{a}\right)}{\partial q_{b k} \partial \dot{q}_{a j}}=\frac{\partial^{2} \mathscr{L}^{(b)}\left(q ; \dot{q}_{b}\right)}{\partial q_{a j} \partial \dot{q}_{b k}}, a \neq b .
$$

This leads to the following sharpening of the decomposition (4.5): in any nonlinear dependence of $\mathscr{L}^{(a)}$ on $\dot{q}_{a}, q_{b}$ for $b \neq a$ cannot occur; while in any linear dependence of $\mathscr{L}^{(a)}$ on $\dot{q}_{a}$ we have conditions imposed by Eq. (4.16). So if we write"

$$
\mathscr{L}^{(a)}\left(q ; \dot{q}_{a}\right)=\mathscr{L}_{\mathrm{nl}}^{(a)}\left(q_{a}, \dot{q}_{a}\right)+\sum_{j} f_{a j}(q) \dot{q}_{a j}-V^{(a)}(q)
$$

then Eq. (4.16) implies

$$
f_{a j}(q)=\frac{\partial f(q)}{\partial q_{a j}},
$$

and the linear terms in (4.17) can be dropped because they amount to a total time derivative in $\mathscr{L}$. Thus at the end of step II the Lagrangian takes the form

$$
\begin{aligned}
& \mathscr{L}(q ; \dot{q})=\sum_{a} \mathscr{L}_{\mathrm{nl}}^{(a)}\left(q_{a} ; \dot{q}_{a}\right)-V(q), \\
& V(q)=\sum_{a} V^{(a)}(q),
\end{aligned}
$$

where each term $\mathscr{L}_{\mathrm{nl}}^{(a)}$ is either zero or a nonlinear function of $\dot{q}_{a}$. At this stage, the two-form $\omega_{\mathscr{L}}$ has achieved a completely separated form:

$$
\begin{aligned}
& \omega_{\mathscr{L}}=\sum_{a} \omega_{\mathscr{L}}^{(a)} \\
& \omega_{\mathscr{L}}^{(a)}=\sum_{j} d\left(\frac{\partial \mathscr{L}_{\mathrm{n} 1}^{(a)}}{\partial \dot{q}_{a j}}\right) \wedge d q_{a j} .
\end{aligned}
$$

However each (nonzero) $\mathscr{L}_{\text {nl }}^{(a)}$ is arbitrary up to a purely $q_{a}$-dependent function, and the subsequent splitting of $\boldsymbol{V}$ into $V^{(a)}$ is also arbitrary.

Step III. The strategy is similar to step 11: to the result (4.15) of that step we apply $L_{\Delta}$ and $L_{X_{K_{m}}}$ and compare the results. With $a \neq b$ understood, we have first

$\omega_{\mathscr{L}}\left[\left[\Delta, \frac{\partial}{\partial q_{a j}}\right], \frac{\partial}{\partial q_{b k}}\right]+\omega_{\mathscr{L}}\left(\frac{\partial}{\partial q_{a j}},\left[\Delta, \frac{\partial}{\partial q_{b k}}\right]\right)=0$.

Now

$$
\left[\Delta, \frac{\partial}{\partial q_{a j}}\right]=-\sum_{c l} \frac{\partial A_{c l}}{\partial q_{a j}} \frac{\partial}{\partial \dot{q}_{c l}},
$$

and similarly with $a j \rightarrow b k$. Using such expressions in Eq. (4.20) and also (4.4) from step I, we get

$\sum_{l} \omega_{\mathscr{L}}^{(a)}\left[\frac{\partial}{\partial q_{a j}}, \frac{\partial}{\partial \dot{q}_{a l}}\right) \frac{\partial A_{a l}}{\partial q_{b k}}=\sum_{l} \omega_{\mathscr{L}}^{(b)}\left(\frac{\partial}{\partial q_{b k}}, \frac{\partial}{\partial \dot{q}_{b l}}\right) \frac{\partial A_{b l}}{\partial q_{a j}}$.

Next when we apply $\mathrm{L}_{X_{K_{m}}}$ to Eq. (4.15) we get

$$
\begin{aligned}
\omega_{\mathscr{L}}\left[\left[X_{K_{m}}, \frac{\partial}{\partial q_{a j}}\right], \frac{\partial}{\partial q_{b k}}\right] \\
+\omega_{\mathscr{L}}\left(\frac{\partial}{\partial q_{a j}},\left[X_{K_{m}}, \frac{\partial}{\partial q_{b k}}\right]\right)=0 .
\end{aligned}
$$

Now we need a different expression for these commutators than in Eq. (4.11):

$$
\begin{aligned}
{\left[X_{K_{m}}, \frac{\partial}{\partial q_{a j}}\right] } & =\sum_{c}\left[q_{c m} \Delta^{(c)}, \frac{\partial}{\partial q_{a j}}\right] \\
& =-\sum_{c l} q_{c m} \frac{\partial A_{c l}}{\partial q_{a j}} \frac{\partial}{\partial \dot{q}_{c l}}+\cdots
\end{aligned}
$$

Here the omitted terms involve $\partial / \partial q_{a}$ and $\partial / \partial \dot{q}_{a}$ : all such terms make no contribution in Eq. (4.23) since $a \neq b$. We then obtain, using Eq. (4.4),

$$
\begin{aligned}
q_{b m} \sum_{l} \omega_{\mathscr{P}}^{(b)}\left[\frac{\partial}{\partial q_{b k}}, \frac{\partial}{\partial \dot{q}_{b l}}\right) \frac{\partial A_{b l}}{\partial q_{a j}} \\
=q_{a m} \sum_{l} \omega_{\mathscr{L}}^{(a)}\left[\frac{\partial}{\partial q_{a j}}, \frac{\partial}{\partial \dot{q}_{a l}}\right] \frac{\partial A_{a l}}{\partial q_{b k}} .
\end{aligned}
$$


Comparing this with Eq. (4.22) we get the main result of Step 111:

$$
\left(q_{a m}-q_{b m}\right) \sum_{l} \omega_{\mathscr{L}}^{(a)}\left(\frac{\partial}{\partial q_{a j}}, \frac{\partial}{\partial \dot{q}_{a l}}\right) \frac{\partial A_{a l}}{\partial q_{b k}}=0,
$$

1.e.,

$$
\frac{\partial}{\partial q_{b k}} \sum_{l} \omega_{\mathscr{L}}^{(a)}\left[\frac{\partial}{\partial q_{a j}}-\frac{\partial}{\partial \dot{q}_{a l}}\right] A_{a l}=0 \text { for } a \neq b .
$$

Thus this result permits the expression

$$
\sum_{l} \omega_{\mathscr{L}}^{(a)}\left[\frac{\partial}{\partial q_{a j}}, \frac{\partial}{\partial \dot{q}_{a l}}\right] A_{a l}
$$

to depend on $q_{a}, \dot{q}_{a}$, and possibly on $q_{b}$ for $b \neq a$ via $A_{a l}$.

The conclusion (4.26) does not directly give us some conditions on the "potential" $V(q)$ in $\mathscr{L}$, such as for instance the separability of $V$. But this is just what we get when we combine the information so far obtained with the assumption that the dynamical equation (2.16) is soluble all over $T Q$. Indeed that equation now reads, in local coordinates,

$$
-\frac{\partial \mathscr{L}_{\mathrm{nl}}^{(a)}}{\partial q_{a j}}\left(q_{a} ; \dot{q}_{a}\right)+\sum_{k}\left|\frac{\partial^{2} \mathscr{L}_{\mathrm{nl}}^{(a)}\left(q_{a} ; \dot{q}_{a}\right)}{\partial \dot{q}_{a j} \partial \dot{q}_{a k}} A_{a k}+\frac{\partial^{2} \mathscr{L}_{\mathrm{nl}}^{(a)}\left(q_{a} ; \dot{q}_{a}\right)}{\partial \dot{q}_{a j} \partial q_{a k}} \dot{q}_{a k}\right|=-\frac{\partial V(q)}{\partial q_{a j}}
$$

We may now use the facts that the left-hand side has no dependence on $q_{b}$ for $b \neq a$, and that we may treat all $q$ 's and $\dot{q}$ 's as independent since solutions $\boldsymbol{A}_{a j}$ exist all over $\mathbf{T} \boldsymbol{Q}$, to conclude that indeed $V(q)$ must be separable:

$$
V(q)=\sum_{a} V^{(a)}\left(q_{a}\right)
$$

Thus we have arrived at a completely separated form for 2:

$$
\mathscr{L}(q ; \dot{q})=\sum_{a} \mathscr{L}_{\mathrm{nl}}^{(a)}\left(q_{a} ; \dot{q}_{a}\right) \rightarrow V^{(a)}\left(q_{a}\right)
$$

Let us stress that this form for $\mathscr{L}$ has not assumed that it is nonsingular. If it is nonsingular then we have proved the no-interaction theorem, since Eq. (4.28) shows that each particle moves independently of the others. It is a simple matter to proceed further and establish that, on using all the consequences of Poincaré invariance, each $V^{(a)}$ must vanish and each $\mathscr{L}_{\text {nl }}^{(a)}$ must take the form"

$$
\mathscr{L}_{\mathrm{nl}}^{(a)}\left(q_{a} ; \dot{q}_{a}\right)=-m_{a}\left[1-\sum_{j} \dot{q}_{a j} \dot{q}_{a j}\right]^{1 / 2}
$$

If the Lagrangian is singular, $A_{a j}$ is determined up to the addition of elements from the (vertical)kernel of $\omega_{\mathscr{L}}^{(a)}$. Equation (4.28) by itself can give no further information about such pieces in $\boldsymbol{A}_{a j}$, and in fact we can imagine that they contain interactions with other particles. However, this cannot happen, as the following analysis shows. From the separable form of the rotation generator $X_{J_{m}}$, Eq. (2.20b), and of the two-form $\omega_{\mathscr{L}}$, Eq. (4.19), it follows that because of the vanishing of $I_{X_{J_{m}}} \omega_{\mathscr{L}}$ we have for each particle

$$
\mathrm{L}_{X J_{m}^{(a)}} \omega_{\mathscr{L}}^{(a)}=0
$$

where

$$
X_{J_{m}}^{(a)}=-\sum_{k l} \epsilon_{k l m}\left[q_{a k} \frac{\partial}{\partial q_{a l}}+q_{a k} \frac{\partial}{\partial \dot{q}_{a l}}\right]
$$

$$
\begin{aligned}
& \omega_{\mathscr{L}}^{(a)}=\sum_{j k}\left[\frac{\partial^{2} \mathscr{L}_{\mathrm{nl}}^{(a)}}{\partial \dot{q}_{a j} \partial \dot{q}_{a k}} d \dot{q}_{a j} \wedge d q_{a k}\right. \\
&+\frac{1}{2}\left[\frac{\partial^{2} \mathscr{L}_{\mathrm{nl}}^{(a)}}{\partial q_{a j} \partial \dot{q}_{a k}}\right. \\
&\left.\left.\quad-\frac{\partial^{2} \mathscr{L}_{\mathrm{nl}}^{(a)}}{\partial q_{a k} \partial \dot{q}_{a j}}\right] d q_{a j} \wedge d q_{a k}\right] .
\end{aligned}
$$

The vertical kernel of $\omega_{\mathscr{L}}^{(a)}$ is determined by the null eigenvectors of the matrix

$$
\left[\frac{\partial^{2} \mathscr{L}_{\mathrm{nl}}^{(a)}}{\partial \dot{q}_{a j} \partial \dot{q}_{a k}}\right] \text {. }
$$

Because of the manifest rotational invariance implied by Eqs. (4.32) and (4.33a), and because the rotation group acts transitively on the set of directions in space, we see that we have only two options: either (i) $\mathscr{L}_{\text {nl }}^{(a)}$ is not identically zero, the matrix

$$
\left(\frac{\partial^{2} \mathscr{L}_{\mathrm{nl}}^{(a)}}{\partial \dot{q}_{a j} \partial \dot{q}_{a k}}\right)
$$

is nonsingular and so $\omega_{\mathscr{L}}^{(a)}$ is nondegenerate; or (ii) $\mathscr{L}_{\text {nl }}^{(a)}$ vanishes identically since it cannot be linear in $\dot{q}_{a}$, and then $\omega_{\mathscr{L}}^{(a)}$ also vanishes identically. In the latter case, combination of translational and rotational invariances easily shows that $q_{a}$ cannot occur in the potential $V(q)$, so all the variables referring to particle "a" are completely absent from 2 . Thus the only sense in which $\mathscr{L}$ could be singular is by virtue of one or more particles, originally included in the enumeration of degrees of freedom, not "showing up" in $\mathscr{L}$ at all. Since this is a trivial situation, we have again established the no-interaction theorem.

\section{CONCLUSION}

The proof given here of the no-interaction theorem is quite economical in comparison with the original as well as more recent proofs in the literature. In arriving at the completely separated form (4.30) for the Lagrangian, only 
the following features played an explicit part in the calculations: (i) the WLC (3.1), (ii) the form (2.20a) for the translation generator $X_{P_{j}}$, (iii) the commutation relation (2.19), and (iv) the annihilation of $\omega_{\mathscr{L}}$ by $L_{\Delta}$ and $L_{X_{K_{m}}}$. Even the successive steps of the calculation in Sec. IV closely resemble one another.

We may point out that the original proof of the theorem for any finite number of particles, which incidentally is rather involved, uses as an essential assumption the possibility of the Hamiltonian being obtainable from a nonsingular Lagrangian. ${ }^{13}$ If this is granted, the proof presented here is much simpler and much more direct than the one based on phase-space methods. On the other hand, in our present approach the separability of the Lagrangian is obtained without invoking its nonsingularity at all. What we do need to assume is that the secondorder dynamics $\mathrm{A}$ does exist everywhere on $\boldsymbol{T Q}$, and not just on some submanifold of $\mathbf{T Q}$. In the terminology of generalized Hamiltonian dynamics, this means that, in addition to the absence of secondary constraints, we require that all the primary constraints be first class. ${ }^{14}$
For the two-particle system, there are Lagrangian models available in the literature, containing both interaction and invariant world lines. ${ }^{15}$ However, in these models, the independent parameter with respect to which the velocities $\dot{q}$ are defined is not physical inertial time, whereas in the present paper it is. For most of the other models treating directly a general number of particles, no Lagrangian is assumed at the outset, and again as in the two-particle case the independent parameter is not ordinary time. There are some attempts in the literature to find a Lagrangian basis for these models, ${ }^{16}$ but it appears somewhat unlikely that in the general case a Lagrangian can be found. The directions in which the work of the present paper may be further examined are then the following: (i) if the independent parameter is not ordinary time but is dynamically determined, and if a Lagrangian exists, then-for instance in the two-particle case-at what point in the present calculations do we see a change permitting interactions and objective world lines to coexist and (ii) what happens if there are some primary secondclass and/or secondary constraints? We hope to come back to these questions elsewhere.
'Permanent address: Centre for Theoretical Studies and Department of Physics, Indian Institute of Science, Bangalore, 560012, India.

1P. A. M. Dirac, Rev. Mod. Phys. 21, 392 (1949).

2D. G. Currie, T. F. Jordan, and E. C. G. Sudarshan, Rev. Mod. Phys. 35, 350 (1963);J. T. Cannon and T. F. Jordan, J. Math. Phys. 5, 299 (1964); H. Leutwyler, Nuovo Cimento 37, 556 (1965); E. C. G. Sudarshan and N. Mukunda, Classical Dynamics-A Modern Perspective (Wiley, New York, 1976).

${ }^{3}$ A. P. Balachandran, G. Marmo, and A. Stern, Nuovo Cimento 69A, 175 (1982); R. Giachetti and E. Sorace, Lett. Nuovo Cimento 26, 1 (1979); J. Martin and J. L. Sanz, J. Math. Phys. 19,780 (1978).

${ }^{4}$ Out of an extensive literature, we give a few references: $D$. Dominici, J. Gomis, and G. Longhi, Nuovo Cimento 48A, 257 (1978); 48B, 152 (1978); T. Takabayashi, Suppl. Prog. Theor. Phys. 67, 1 (1979); A. Komar, Phys. Rev. D 18, 1881 (1978); 18, 1887 (1978); 18, 3017 (1978); 19, 2908 (1979); I. T. Todorov, in Proceedings of the Istanbul Lectures, August, 1979 (unpublished); in proceedings of the Karpacz Lectures, 1980 (unpublished); F. Rohrlich, Ann. Phys. (N.Y.) 117, 292 (1979); Physica A96, 290 (1979); N. Mukunda and E. C. G. Sudarshan, Phys. Rev. D 23, 2210 (1981); E. C. G. Sudarshan, N. Mukunda, and J. N. Goldberg, ibid. 23, 2218 (1981); A. P. Balachandran, G. Marmo, N. Mukunda, J. S.
Nilsson, A. Simoni, E. C. G. Sudarshan, and F. Zaccaria, Nuovo Cimento 67A, 121 (1982); J. Math. Phys. 25, 167 (1984).

5J. Samuel, Phys. Rev. D 26, 3475 (1982);26, 3482 (1982).

6 We refer to R. Abraham and J. E. Marsden, Foundations of Mechanics (Addison-Wesley, Reading, Mass., 1978), for the theory and notation of calculus on manifolds.

${ }^{7}$ See, in this connection, R. Jost, Rev. Mod. Phys. 36, 572 (1964).

${ }^{8} \mathrm{This}$ is appropriate to the fact that we are using ordinary time as the independent parameter - the instant description.

${ }^{9}$ For a detailed discussion see, for instance, Sudarshan and $\mathrm{Mu}$ kunda (Ref. 2).

${ }^{10}$ This conclusion is very similar in form to an analogous conclusion in the phase-space proof of H. Leutwyler (Ref. 2).

${ }^{11}$ The subscript nl means nonlinear with respect to $\dot{q}_{a}$.

${ }^{12}$ See, for example, Balachandran, Marmo, and Stern (Ref.3).

${ }^{13}$ This assumption, implicit in Leutwyler (Ref. 2), has been emphasized in Sudarshan and Mukunda (Ref. 2).

${ }^{14}$ The reason for this connection is explained in G. Marmo, N. Mukunda, and J. Samuel, Riv. Nuovo Cimento 6, No. 2, (1983).

${ }^{15}$ Dominici, Gomis, and Longhi (Ref. 4).

16J. Gomis, K. Kamimura and J. M. Pons, Firenze report (unpublished). 